\title{
Comparação entre os valores teóricos para alguns dados espirométricos em crianças determinados pelas equações de Mallozi e de Polgar*
}

\author{
Waldemar Ladosky¹, Rogerson T. Andrade², Noel Guedes loureiro², Marcos Alberto Machado Botelho ${ }^{3}$
}

Objetivo: 0 presente trabalho dá prosseguimento ao estudo da validade de equações para valores teóricos (VT) para cálculos espirométricos na população brasileira. Foi feita a comparação entre as equações propostas por Mallozzi (VT-M) para população brasileira e as de Polgar (VT-P), utilizadas especialmente na Europa e Estados Unidos. Métodos: Foram analisados os VT medidos em 185 pacientes de ambos os sexos, com idade entre 6 e 17 anos, sendo 89 meninos e 96 meninas. Para cada paciente foi feito o cálculo do VT para $C V F, V E F_{1}$ e $F_{E F} F_{25-75}$ segundo as equações de Mallozi VT-M e de Polgar VT-P. Em seguida foram comparados os laudos obtidos por um e por outro, segundo 0 Consenso de Espirometria da SBPT. Resultados: O VT-M para CVF é 0,38\% maior para os meninos e $2,19 \%$ para as meninas que 0 calculado segundo Polgar. Os VT-P para 0 VEF ${ }_{1}$ são $6,67 \%(p<0,05)$ menores para as meninas e $7,26 \%$ para os meninos. Para $0 \mathrm{FEF}_{25-75}$ os valores diferem apenas em 0,35 e 0,43\% para cada um dos sexos, sem significância estatística. Conclusão: Os VTs de CVF e $\mathrm{VEF}_{1}$ para crianças brasileiras, avaliados segundo Mallozzi, diferem significativamente quanto aos VTs de Polgar. Como conseqüência, encontramos, utilizando as equações de Mallozzi, tendência maior em diagnosticar dificuldade obstrutiva e menor em achados de insuficiência ventilatória restritiva.

(J Pneumol 2002;28(3):125-130)

\section{Comparison between the theoretical values for spirometric data} in children determined by Mallozi's and Polgar's equations

Objective: This paper is a continuation of the study on the validity of equations of predicted value (PV) for the Brazilian population. A comparison was made between the equations proposed by Mallozi and by Polgar for the Brazilian population, which are mainly used in the United States and Europe. Methods: PVs of 185 patients of both sexes ( 89 boys and 96 girls), ages between 6 and 17 years, were analyzed. For each subject, the PVs for forced vital capacity (FVC), forced expiratory volume at $1 \mathrm{sec}$. $\left(\mathrm{FEV}_{1}\right)$, and the medium expiratory flow (MEF) were calculated according to the equations proposed by Mallozi and Polgar. Results were compared. Diagnoses were made according to the Brazilian Consensus of Spirometry. Results: The PV-M for FVC is $0.38 \%$ higher for boys and $2.19 \%$ higher for girls than that determined by Polgar. The PV-P for

FEV $_{1}$ is $6.67 \%(p<0.05)$ lower for girls, suggesting an underassessment of the obstructive impairment. For boys, a mean lower value of $7.26 \%$ as compared to the VT-M is at the limit of significance $(0.05<p<0.01)$. No significant difference between the two equations analyzed was

found for MEF. Conclusions: The PVs for $C V F$ and $\mathrm{VEF}_{1}$ for Brazilian children, according to Mallozzi, are significantly different when compared to those published by authors like Polgar. As

a result, the authors found that Mallozzi's equations are more likely to detect obstructive impairment and less likely to diagnose restrictive ventilatory insufficiency.

\footnotetext{
* Trabalho realizado no Laboratório de Função Pulmonar, Hospital das Clínicas da Universidade Federal de Pernambuco, Recife, PE.

1. Professor Titular.

2. Estudante de Medicina.

3. Professor Adjunto.
}

Endereço para correspondência - Av. Prof. Morais Rego, s/no, Cidade Universitária - 50670-420 - Recife, PE. Fax (81) 3271-8534. Email: doskyla@hotlink.com.br

Recebido para publicação em 9/9/01. Aprovado, após revisão, em 13/3/02. 
Descritores - Espirometria. Capacidade vital. Volume expiratório forçado. Fluxo máximo médio expiratório. Estudos de casos e controles. Modelos teóricos.

Key words - Spirometry. Vital capacity. Forced expiratory volume. Maximal medium expiratory flow rate. Case-control studies. Theoretical models.

\section{INTRODUÇÃO}

Binder et al.(1) estudaram a função pulmonar, especialmente as medidas da capacidade vital forçada (CVF) e 0 volume expiratório forçado de 1 seg. $\left(V_{E F}\right)$ em crianças brancas e negras entre 9 e 17 anos. Observaram que os parâmetros diferiam entre si de acordo com a raça e, dentro da mesma raça, evoluíam com a idade e altura. Outros trabalhos confirmaram o dimorfismo sexual para a CVF, para o $\mathrm{VEF}_{1}$ e para o fluxo expiratório forçado entre 25 e $75 \%\left(\mathrm{FEF}_{25-75}\right)$, sendo os valores para meninos sistematicamente superiores aos de meninas ${ }^{(2-8)}$.

Há concordância quanto ao aumento da CVF e do $\mathrm{VEF}_{1}$ com a idade $\mathrm{e}^{(1,2,4,5,9,10-14)}$. Os valores crescem com a idade até os 17 ou 18 anos $^{(2,12)}$, quando atingem um platô e começam a decair. $O$ achado que crianças caucasianas apresentam valores de CVF e VEF 1 superiores aos das negras de mesma idade tem sido descrito de maneira consistente ${ }^{(1,3,5-8,10)}$. H su et al. ${ }^{(15)}$ estudaram crianças americanas pertencentes aos grupos branco, negro e méxicoamericano e encontraram para estes últimos valores significativamente menores que os do grupo branco e superiores aos do negro. Chinn e Rona ${ }^{(13)}$ estudaram, em Londres, crianças caucasianas, negras de origem caribenha e hindus e observaram que os valores de CVF, $\mathrm{VEF}_{1}$ e $\mathrm{FEF}_{25-75}$ eram mais elevados para os brancos, seguidos dos caribenhos e, finalmente, dos negros. No mesmo trabalho os autores compararam crianças inglesas com escocesas residentes na cidade de Londres e encontraram valores significativamente mais elevados para estas, o que mostra que pode haver variação dentro de um mesmo grupo étnico, o que reforça a idéia de Schoenberg et al. ${ }^{(5)}$ de que as variações encontradas entre os diversos grupos étnicos são devidas a fatores genéticos.

As variações dos parâmetros expiratórios em crianças levou à criação de equações de "valor teórico" (VT) que os normatizassem, tornando as comparações possíveis(15), e permitissem assim diferenciar função respiratória normal de alterada. Polgar e Weng(16) realizaram extensa revisão das equações existentes e propuseram as mais coerentes para uso clínico em crianças, que são usualmente conhecidas como "equações" de Polgar, largamente utilizadas em nosso meio.

Pereira et al.(17), baseados em uma amostra significativa da população adulta brasileira, propuseram equações que são basicamente diferentes das americanas e euro-
Siglas e abreviaturas utilizadas neste trabalho

VT - Valores teóricos

VT-M - Valor teórico de Mallozi

VT-P - Valor teórico de Polgar

CVF - Capacidade vital forçada

$V E F_{1}$ - Volume expiratório forçado de $1 \mathrm{seg}$.

$\mathrm{FEF}_{25-75}$ - Fluxo expiratório forçado entre $25-75 \%$

SBPT - Sociedade Brasileira de Pneumologia e Tisiologia

OMS - Organização Mundial de Saúde

EBEM - Empresa Brasileira de Engenharia Médica

ATS - American Thoracic Society

CECA - Communauté Européenne du Charbon et de l'Acier

péias. Mallozi(18), após levantamento de população infantil em São Paulo, propôs equações de VT que expressassem o padrão normal para uma população miscigenada e, portanto, geneticamente diferenciada, como a brasileira.

Este estudo propõe-se a comparar, para uma mesma criança, os valores teóricos obtidos pela aplicação das equações de Polgar com os obtidos com as equações de Mallozi, e observar sua importância para o diagnóstico.

\section{Material e métodos}

\section{Pacientes}

Foram analisados espirogramas de 185 crianças, de ambos os sexos (89 meninos e 96 meninas), com idade entre 6 e 17 anos, com altura menor que $170 \mathrm{~cm}$, que vieram de rotina ao Serviço, enviados pelos ambulatórios de Pediatria do Hospital das Clínicas e do Hospital Barão de Lucena, para avaliação da função pulmonar. Não foram levados em conta os diagnósticos prévios dos pacientes.

Em consonância com normas da OMS, os grupos foram divididos em pré-púberes (entre seis e 10 anos de idade; perpúberes (10 anos e um mês a 14 anos) e póspúberes (idade entre 14 anos e um mês e 17 anos). Foram excluídos pacientes de qualquer idade com altura igual ou superior a $170 \mathrm{~cm}$, medida com pé descalço. A distribuição por sexo e a pelas faixas etárias estão descritas na Tabela 1.

Não foi excluída qualquer patologia e nenhum dos pacientes havia adquirido o hábito do tabagismo.

TABELA 1

Distribuição dos pacientes segundo grupo etário e sexo

\begin{tabular}{lcc}
\multicolumn{1}{c}{ Idade } & Meninos & Meninas \\
\hline$<10$ anos & 30 & 19 \\
$>10<13$ anos 11 meses & 37 & 31 \\
$>14<16$ anos 11 meses & 22 & 46 \\
Total & 89 & 96 \\
\hline
\end{tabular}




\section{Espirogramas}

Para cada paciente foi realizada prova de espirometria forçada dentro das normais previstas pelo Consenso Brasileiro de Espirometria ${ }^{(19)}$, sendo a manobra realizada segundo D'Angelo et al.(20). Não foram incluídos no estudo os espirogramas que apresentaram volume extrapolado $>5 \%$ da CVF ou que possuíssem indícios de má execução, como finalização precoce, fechamento de glote, tosse, etc. Os pacientes pouco cooperativos foram também excluídos.

Para cada espirograma foi feito o cálculo do valor teórico segundo as equações de Polgar ${ }^{(16)}$ e de Mallozi ${ }^{(18)}$ para CVF, VEF e $F E F_{25-75}$ e os resultados comparados por grupos etários. 0 valor espirométrico, obtido para cada um dos parâmetros em estudo, foi avaliado em relação a cada valor teórico. Foi então estabelecido laudo, para cada par de resultados decorrentes de um mesmo esforço, segundo o Consenso Brasileiro de Espirometria.

Foi tomado o quinto percentil como limite para normalidade, abaixo do qual foram estabelecidos os diagnósticos de distúrbio obstrutivo, restritivo ou misto, conforme o caso.

\section{Equipamento}

Foi utilizado um espirômetro fluxométrico Beatrice da EBEM (Recife-Brasil), que utiliza um pneumotacômetro Fleisch IV acoplado a um transdutor de pressão diferencial e que atende às normas da SBPT, ATS e CECA. $O$ aparelho era calibrado toda manhã utilizando uma seringa de três litros, fazendo-se três fluxos distintos, ajustando-se para umidade, temperatura e pressão atmosférica.

As provas eram realizadas invariavelmente pela manhã, com a sala mantida a $25^{\circ} \mathrm{C}$ e com o paciente sentado. 0 paciente era solicitado a realizar pelo menos três manobras, sendo selecionada a que apresentasse melhor soma de $C V F+V E F_{1}$, e não diferissem entre si em mais de $100 \mathrm{ml}$ ou $5 \%$.

0 programa utilizado (Pulmosoft 4.0 - 1998) permite, por meio de seleção em menu, o cálculo automático, para cada paciente, do valor teórico, tanto segundo as equações de Polgar(16) como as de Mallozzi ${ }^{(18)}$.

\section{Estatística}

Para cada faixa etária foram inicialmente calculadas as médias aritméticas e os desvios padrões para os VTs de cada parâmetro ( $C V F, V F_{1}, \mathrm{FEF}_{25-75}$ ) obtido segundo Mallozi e Polgar, para cada faixa etária. Em seguida foi feita a medida das diferença entre as médias dos valores teóricos correspondentes (Polgar e Mallozi). Procedeu-se então à comparação estatística entre as médias de cada parâmetro pelo teste $t$ de Student.

Tomando-se as equações de Mallozi(18) como padrão, por terem sido estabelecidas em amostra de população brasileira, foram analisadas as coincidências e divergências de laudo para cada paciente e os resultados tabelados. Foi considerado como limite de significância $p<0,05$.

\section{Resultados}

Diferença entre as médias dos VTs

a) Grupo pré-púbere (Figura $1 \mathrm{~A}$ e $\mathrm{B}$ )

Nesse grupo a média dos valores teóricos para CVF, ainda que discretamente mais elevados no sexo masculino, são indistintos, qualquer uma das equações seja utilizada.

Talvez devido ao número pequeno da amostra, para sexo masculino, ainda que $0 \mathrm{VT}$ do $\mathrm{VEF}_{1}$ seja $100 \mathrm{ml}$ mais elevado, a diferença não tem significância estatística. Nas meninas, entretanto, a diferença é de $170 \mathrm{ml}$ e é estatisticamente significativa.

$O \mathrm{FEF}_{25-75}$ em pré-púberes é mais elevado quando medido por Mallozi, qualquer que seja o sexo. As diferenças são não significativas para os meninos e se encontram no limite da significância para as meninas.

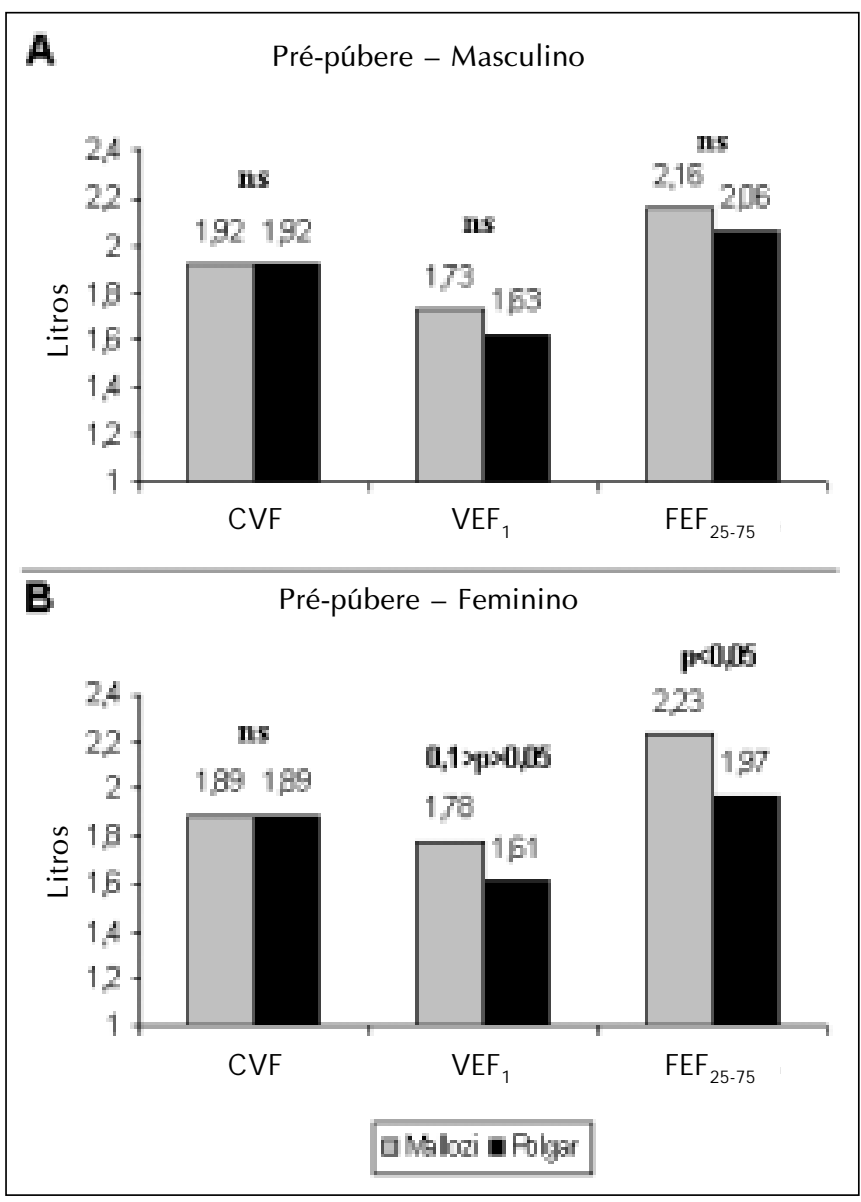

Figura 1 - Comparação entre a média das variáveis estudadas no grupo de crianças pré-púberes do sexo masculino $(\boldsymbol{A})$ e do sexo feminino (B) 
b) Fase perpúbere

A exemplo do grupo anterior, a média doS VT-M e VT-P para CVF foi idêntica para ambos os sexos.

OS VT-M para $\mathrm{VEF}_{1}$ foram, nessa faixa etária, significativamente mais elevados que os VT-P, tanto entre os meninos $(p<0,02)$ como entre as meninas $(p<0,015)$.

Os valores de $\mathrm{FEF}_{25-75}$ são superiores aos do grupo prépúbere, mas não diferem entre si nem quanto ao sexo nem quanto à equação utilizada.

c) Fase pós-púbere (Figura $3 \mathrm{~A}$ e B)

Os três parâmetros analisados elevam-se dramaticamente com a puberdade, sendo sempre superiores para os meninos. As equações, tanto de Mallozzi como as de Polgar, levaram, em ambos os sexos, a valores médios de CVF e $\mathrm{FEF}_{25-75}$, não são significativamente diferentes.

As médias para $0 \mathrm{VEF}_{1}$ também se elevam de maneira significativa depois da puberdade e são mais elevadas nos meninos que nas meninas. A diferença em relação às de Polgar foi, entretanto, significativa apenas entre as meninas $(p<0,015)$. Ainda que a diferença para os meninos

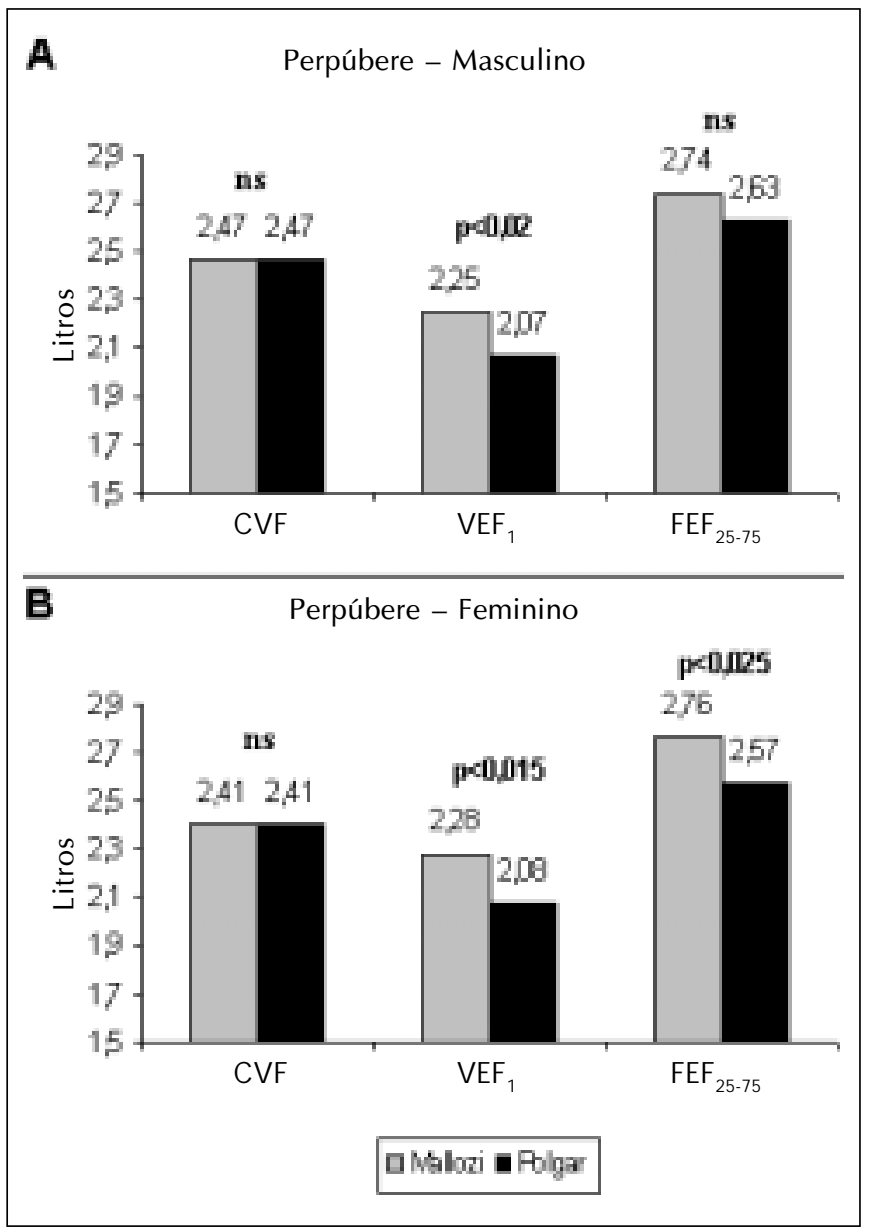

Figura 2 - Comparação entre a média das variáveis estudadas no grupo de crianças perpúberes do sexo masculino $(\boldsymbol{A})$ e do sexo feminino (B) medido por Mallozi e por Polgar seja de $210 \mathrm{ml}$, esta, talvez devido ao pequeno tamanho da amostra, não é significativa.

Variação de diagnóstico segundo a VT utilizada

\section{a) Grupo pré-púbere}

Para pacientes menores de 10 anos de idade, do sexo masculino, o fato de oS VTs para CVF, VEF ${ }_{1}, \mathrm{e} \mathrm{FEF}_{25-75}$ não diferirem significativamente segundo a equação utilizada levou a uma absoluta concordância nos diagnósticos de "normal", "obstrutivo", "restritivo "e "misto".

Para as meninas, na mesma faixa etária, o valor de VTP para $\mathrm{VEF}_{1}$ é menor que 0 VT-M, 0 que levou a discordância em $100 \%$ dos "obstrutivos" identificados por Mallozi, que são classificados como "normais". Os demais diagnósticos coincidem.

\section{b) Fase perpúbere}

Para os indivíduos na faixa da puberdade, o único desacordo encontrado foi quanto ao diagnóstico de "obstrutivo". Para as meninas houve significativo desacordo
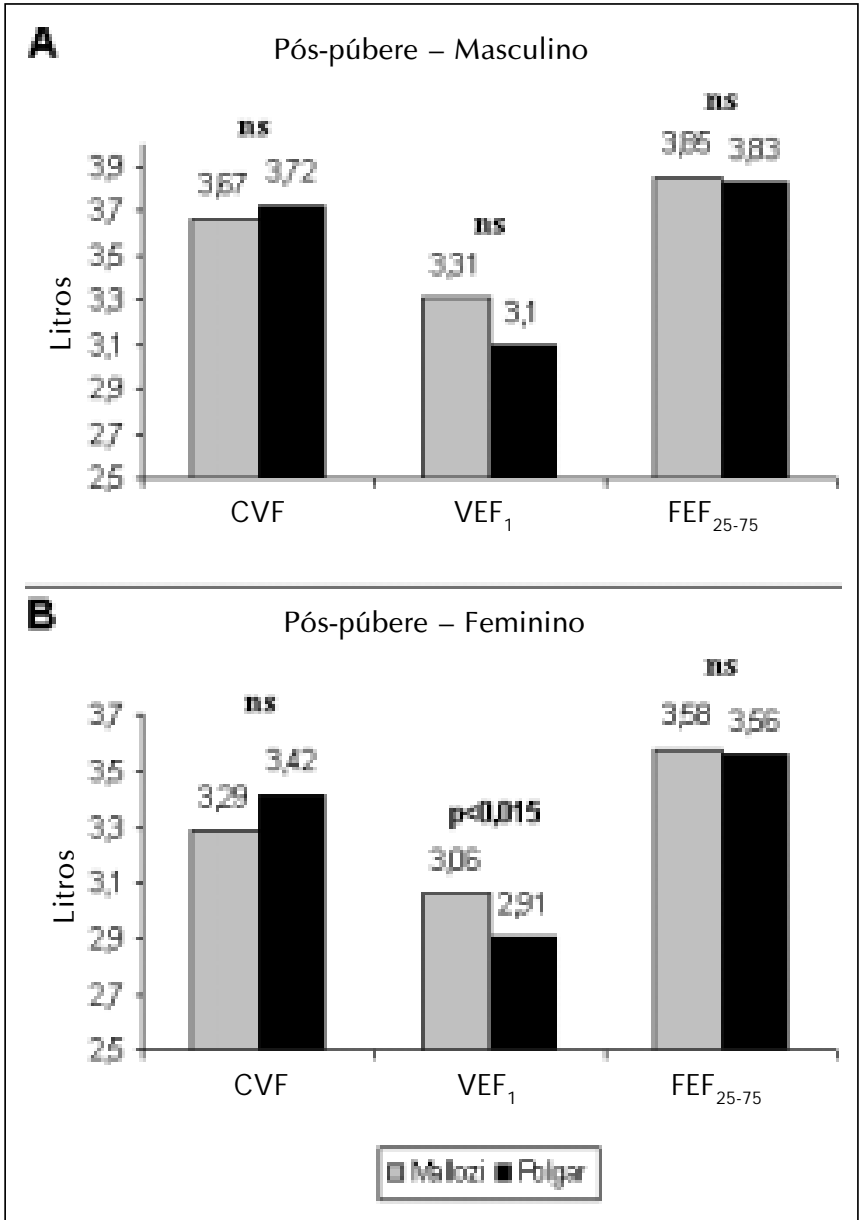

Figura 3 - Comparação entre a média das variáveis estudadas no grupo de crianças pós-púberes do sexo masculino $(\boldsymbol{A})$ e do sexo feminino (B) 
quanto ao diagnóstico de distúrbio "obstrutivo", fato este devido provavelmente à significância da diferença encontrada entre oS VTs de VEF e FEF $_{25-75}$. Para sete pacientes do sexo feminino, identificados como "obstrutivos" pela equação de Mallozi, Polgar considerou quatro "normais" e um "restritivo", discrepância de 71,4\%.

Para os meninos, Polgar levou à discordância em três de seis (50\%) "obstrutivos", sendo os demais diagnósticos coincidentes.

c) Fase pós-púbere

Meninos

Como visto na Figura 3A, os valores de VT-M e VT-P para CVF, em meninos, não diferem entre si significativamente; como resultado, não há diferenças nos diagnósticos de "normal", restritivo" ou "misto", estabelecidos pelo uso de qualquer das duas equações estudadas (Tabela 2).

Apesar de os valores médios de VT-M e VT-P para VEF apresentarem diferença de $210 \mathrm{ml}$, esta não é estatisticamente significativa, talvez devido ao pequeno tamanho da amostra. $\mathrm{Na}$ prática, entretanto (Tabela 2), ela leva a discordância de $33,3 \%$ nos diagnósticos de distúrbio "obstrutivo". Novamente, é importante salientar o número pequeno da amostra.

$\mathrm{O}$ fato de que os VTs para $\mathrm{FEF}_{25-75}$ não diferem entre si mostra que, nesses casos pelo menos, eles não contribuem para o diagnóstico de distúrbio respiratório.

TABELA 2

Comparação dos diagnósticos obtidos em espirometria feita em meninos púberes utilizando as equações para valor teórico de Mallozi e de Polgar

\begin{tabular}{lcccc}
\hline $\begin{array}{c}\text { Laudo } \\
\text { Mallozi }\end{array}$ & $\begin{array}{c}\text { № } \\
\text { pacientes }\end{array}$ & $P \neq M$ & $\begin{array}{c}\% \\
\text { diferença }\end{array}$ & $\begin{array}{c}\text { Laudo } \\
\text { Polgar }\end{array}$ \\
\hline Normal & 11 & 0 & 0 & \\
O bstrutivo & 3 & 1 & 33,3 & Restritivo \\
Restritivo & 7 & 0 & & \\
Misto & 1 & 0 & & \\
\hline
\end{tabular}

TABELA 3

Comparação dos diagnósticos obtidos em espirometria feita em meninas púberes utilizando as equações para valor teórico de Mallozi e de Polgar

\begin{tabular}{lcccl}
$\begin{array}{c}\text { Laudo } \\
\text { Mallozi }\end{array}$ & $\begin{array}{c}\text { № } \\
\text { pacientes }\end{array}$ & $P \neq M$ & $\begin{array}{c}\% \\
\text { diferença }\end{array}$ & $\begin{array}{c}\text { Laudo } \\
\text { Polgar }\end{array}$ \\
\hline Normal & 18 & 3 & 16,7 & 3 restritivos \\
O bstrutivo & 5 & 5 & 100 & 3 normais, 2 mistos \\
Restritivo & 22 & 0 & 0 & \\
Misto & 1 & 1 & 100 & Restritivo \\
\hline
\end{tabular}

Meninas

Entre as meninas pós-púberes (Figura 3B), o valor significativamente mais elevado da VT-M para VEF ${ }_{1}$ levou a maior detecção de distúrbio "obstrutivo", do que pelo uso do VT-P (Tabela 2). Assim, nos cinco casos diagnosticados como "obstrutivos", Polgar encontrou três normais e em dois outros sobrevalorizou uma redução da CVF e classificou como "misto". O único caso identificado como "misto" por Mallozi foi classificado como "restritivo" pelo Polgar.

Como se vê na Figura 3B, o vT-P para CVF é $130 \mathrm{ml}$ mais elevado que o VT-M, não sendo significativa. Como resultado, os diagnósticos de distúrbio "restritivo" são concordantes, qualquer que seja a equação utilizada. Em alguns poucos casos, entretanto, essa diferença é suficiente para o VT-P identificar uma "restrição" quando o VT-M considera normal, como em 3/18 (16,7\%); ou um outro "misto", quando Polgar classifica apenas como "restritivo".

\section{DISCUSSÃo}

Os presentes resultados confirmam prévias publicações ${ }^{(2,4,5,7,10-14)}$ que mostram que entre os três e 24 anos a função respiratória cresce com a idade. Discordam, entretanto, de Seely et al. ${ }^{(4)}$, que descrevem estabilização da CVF em meninas aos 14 anos, enquanto nós encontramos aumento significativo, em ambos os sexos, no grupo de maiores de 14 anos (Figura 3).

Binder et al.(1) e Dockery et al.(10), estudando valores de CVF e VEF ${ }_{1}$, descrevem que estes são superiores para 0 sexo masculino quando comparados com meninas da mesma raça entre as idades de nove e 17 anos. Esses resultados são por nós confirmados para qualquer que tenha sido a equação utilizada. Para $0 \mathrm{VEF}_{1}$ concordamos que os valores para meninos sejam superiores que os encontrados para meninas, ainda que seja a significância discutível devido ao pequeno número da amostra.

Nossos resultados concordam com os de Dickman et al. ${ }^{(2)}$, de Taussing et al. ${ }^{(9)}$ e de Knudson et al. ${ }^{(11)}$, que mostram que até os 12 anos os valores de CVF, VEF ${\text { e } F E F_{25-75}}$ são semelhantes para os dois sexos, passando a se diferenciar na puberdade. Chehreh et al. (3), Schwart et al. ${ }^{(6)}$, Chinn e Rona(13) e Rosenthal et al. ${ }^{(14)}$ descrevem valores distintos para CVF e VEF ${ }_{1}$ em crianças, sendo mais elevados em meninos.

A base fundamental no dimorfismo sexual descrito por esses autores e a ausência, especialmente para a CVF, de diferença entre os sexos para indivíduos entre seis e 17 anos deve estar ligado a um fator genético, como sugerido por Chehren et al. ${ }^{(3)}$ e diversos outros autores $(1,5-8,13)$. Deve ser considerado que Mallozi estudou uma pequena amostra de população de São Paulo que, apesar do forte 
contingente europeu, incorpora significativamente elementos asiáticos e africanos. Binder et al. (1) trabalharam com dois, bem diferenciados, de brancos e negros, da mesma forma que todos os demais autores citados, com exceção de Chinn e Rona(13), que compararam isolados de ingleses com de hindus e de africanos.

Polgar e Weng ${ }^{(16)}$ analisaram uma população do Michigan, de características raciais nitidamente caucasianas, enquanto Mallozi(18) estudou população multirracial em São Paulo. É de chamar atenção, portanto, que, trabalhando com dois isolados distintos, os dois autores tenham encontrado resultados comparáveis em todas as faixas etárias e em ambos os sexos. Esses resultados discrepam

\section{REFERÊNCIAS}

1. Binder RE, Mitchell CA, Schoenberg JB, Bouhuys A. Lung function among black and white children. Am Rev Respir Dis 1976;114:9559.

2. Dickman ML, Schmidt CD, Gardner RM. Spirometric standards for normal children and adolescents (ages 5 years through 18 years). Am Rev Respir Dis 1971;104:680-7.

3. Chehreh MN, Young Jr. RC, Viane H, Ross CW, Scott RB. Spirometric standards for healthy inner-city black children. Am J Dis Child 1973; 126:159-63

4. Seely JE, Guzman CA, Beclake MR. Heart and lung function at rest and during exercise in adolescence. J Appl Physiol 1974;36:34-40.

5. Schoenberg JB, Beck GL, Bouhuys A. Growth and decay of pulmonary function in healthy blacks and whites. Respir Physiol 1978;33: 367-93.

6. Schwartz J, Katz SA, Fegley RW, Tockman MS. Sex and race differences in the development of lung function. Am Rev Respir Dis 1988; 136:1415-21.

7. Enright PL, Linn WS, Avol EL, Margolis HG, Gong Jr. H, Peters J M. Quality of spirometry test performance in children and adolescents. Experience in a large field study. Chest 2000;118:665-71.

8. Joseph CLM, Ownby DR, Peterson EL, J ohnson CC. Racial differences in physiologic parameters related to asthma among middle-class children. Chest 2000;117:1336-44.

9. Taussing LM, Cota K, Kaltenborn W. Different mechanical properties of the lung in boys and girls. Am Rev Respir Dis 1961;123:640-3.

10. Dockery DW, Berkey CS, Ware JH, Speizer FE, Ferris J r. BG. Distribution of forced vital capacity and forced expiratory volume in one second in children 6 to 11 years of age. Am Rev Respir Dis 1983;128: 405-12. dos de Chinn e Rona ${ }^{(13)}$, que encontraram diferença entre crianças inglesas e escocesas e, na mesma cidade de Londres, entre 0 isolado de brancos e o de hindus.

As diferenças significativas encontradas para $0 \mathrm{VEF}_{1}$ com importante repercussão nos laudos em meninas (Tabela 3).

Concluindo, não há diferença, para a confirmação de uma dificuldade "restritiva", na utilização de qualquer uma das equações para VT estudadas. 0 uso da equação de Mallozzi para o $\mathrm{VEF}_{1}$, entretanto, é mais sensível para identificar dificuldade "obstrutiva" que a de Polgar. Acreditamos que, por traduzir melhor a realidade brasileira, deva ser utilizada como equação de escolha.

11. Knudson RJ, Lebowitz MD, Holberg CJ, Burrows B. Changes in the normal maximal expiratory flow-volume curve with growth and aging. Am Rev Respir Dis 1983;127:725-34.

12. Lebowitz MD, Holberg CJ, Knudson RJ , Burrows B. Longitudinal study of pulmonary function development in childhood, adolescence and early adulthood. Am Rev Respir Dis 1987;136:69-75.

13. Chinn SE, Rona RJ. Height and age adjustment for cross sectional studies of lung function in children aged 6-11 years. Thorax 1992;47: 707-14.

14. Rosenthal M, Bain SH, Cramer D, Helms P, Denison D, Bush A, et al. Lung function in white children aged 4 to 19 years: I-Spirometry. Tho$\operatorname{rax} 1993 ; 48: 704-802$

15. Hsu KHK, J enkins DE, H si BP, Bourhofer E, Thompson V, Tanakawa $N$, et al. Ventilatory functions of normal children and young adults, Mexican-American, white and black. Part I Spirometry. J Pediatr 1979; 95:14-23

16. Polgar $C$, Weng TR. The functional development of the respiratory system. Am Rev Respir Dis 1979;120:625-95.

17. Pereira CAC, Barreto SP, Simões J G, Pereira FWL, Gerstler J G, Nakamura J. Valores de referência para espirometria em uma amostra da população brasileira. J Pneumol 1992;18:10-22.

18. Mallozi MC. Valores de referência para espirometria em crianças e adolescentes, calculados a partir de uma amostra da cidade de São Paulo [Tese]. São Paulo: Escola Paulista de Medicina, 1995.

19. SBPT. I Consenso Brasileiro de Espirometria. J Pneumol 1966;22: 105-64.

20. D'Angelo E, Prandi E, Marazzini L, Milic-Emili J. Dependence of maximal flow-volume curves on time course of preceding inspiration in patients with chronic obstructive pulmonary disease. Am J Respir Crit Care Med 1994;150:1581-6. 